\title{
Interpretation of High Resolution Scanning Transmission Electron Microscope Images Based on Core Loss Spectroscopy.
}

\author{
M.P. Oxley, ${ }^{*}$ S.D. Findlay,** and L.J. Allen** \\ * Condensed Matter Sciences Division, Oak Ridge National Laboratory, PO Box 2008, Oak Ridge, \\ TN 37831-6064 \\ ** School of Physics, The University of Melbourne, Victoria 3010, Australia
}

Many factors contribute to the formation of scanning transmission electron microscope (STEM) images, leading to results that are sometimes non-intuitive. These factors include the propagation of the probe through the sample, the localization of the interaction on which the imaging is based and whether the interaction can be realistically described by a "local" or "object function" approximation. The development of aberration correctors for the probe forming optics [1] has led to sub-Ångstrom probes. Such probes are more readily scattered from the atomic columns in crystalline samples than broader probes which tend to "channel" along the atomic columns. This results in an enhanced signal from electrons that are "dechanneled" via ineleastic scattering, primarily thermal diffuse scattering (TDS). Quantitative interpretation of high resolution STEM images requires that this contribution be correctly modeled. For annular dark field (ADF) this has been most effectively handled using the frozen phonon model [2]. We present a frozen phonon model which allows the simulation of STEM images based on electron energy loss spectroscopy (EELS) [3]. This mixed dynamical form factor (MDFF) approach correctly accounts not only for the delocalization of the EELS interaction, but also for the non-local nature of the "effective" scattering potential [4]. We compare this approach to previous formulations and thus illustrate the importance of correctly modeling the contribution to STEM EELS images from all incident electrons, rather just than the "dynamical" electrons included in the more usual Bloch wave/multislice formulation [5].

The importance of the contribution of TDS electrons is illustrated in FIG. 1 where STEM images calculated for the Ag $M$-shell EELS signal are plotted for both the frozen phonon and multislice formulations. Clearly the contribution of TDS electrons (not included in the multislice calculation) is significant for such a heavy scatterer. In addition the peaks in the EELS signal occur not on the atomic columns but between them. We will show how this unexpected behavior may be explained by careful consideration of the channeling conditions.

The non-local nature of the "effective" inelastic scattering potential for EELS is also an important factor in the formation of STEM EELS images [6]. The simulated EELS signal derived from C $K$ shell ionization is shown in FIG. 2. This is for an aberration-free probe formed by $200 \mathrm{keV}$ electrons incident along the [011] zone axis. The line scan is taken through the $\mathrm{Si} / \mathrm{C}$ dumbbells along the [100] direction. Here we see that the signal from the $\mathrm{C} K$-shell is actually larger when the probe is placed above the $\mathrm{Si}$ column than when the probe is on the $\mathrm{C}$ column, despite the $\mathrm{C}$ column effectively not being illuminated when the probe is positioned above the Si Column. We will show that this remarkable phenomenon is due to the non-local nature of the "effective" scattering potential. 
References

[1] N. Dellby et al., J. Electron Microsc. 50 (2001) 177.

[2] S. Hilliard et al., Ultramicroscopy 49 (1993) 14.

[3] S.D. Findlay et al., Ultramicroscopy submitted for publication (2005).

[4] L.J. Allen and T.W. Josefsson, Physical Review B 52 (1995) 3184.

[5] L.J. Allen et al., Ultramicroscopy 96 (2003) 47.

[6] M.P. Oxley et al., manuscript in preparation (2005).

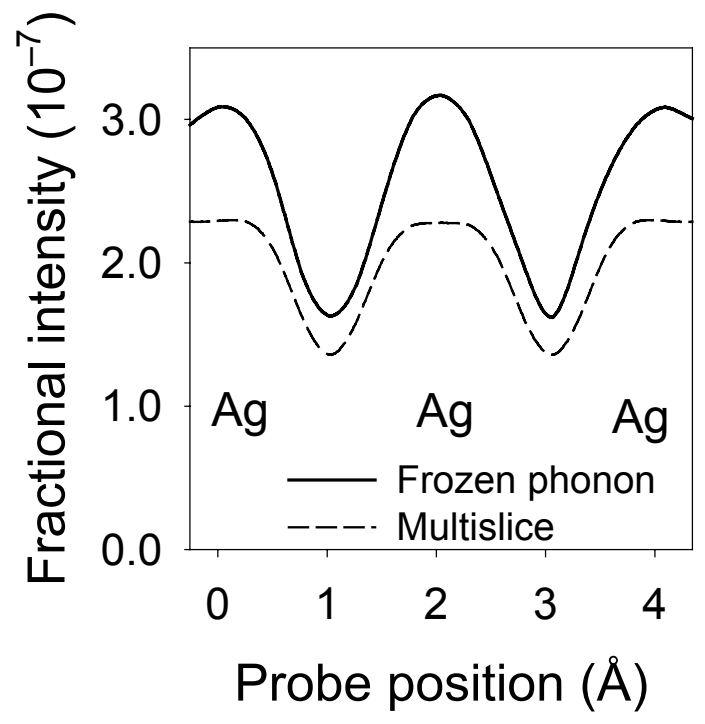

FIG. 1. Ag $M$-shell EELS image calculated for the [001] zone axis conditions and a line scan along the [100] direction. This calculation is for $100 \mathrm{keV}$ incident electrons and an aberration balanced probe obtainable using an aberration corrector.

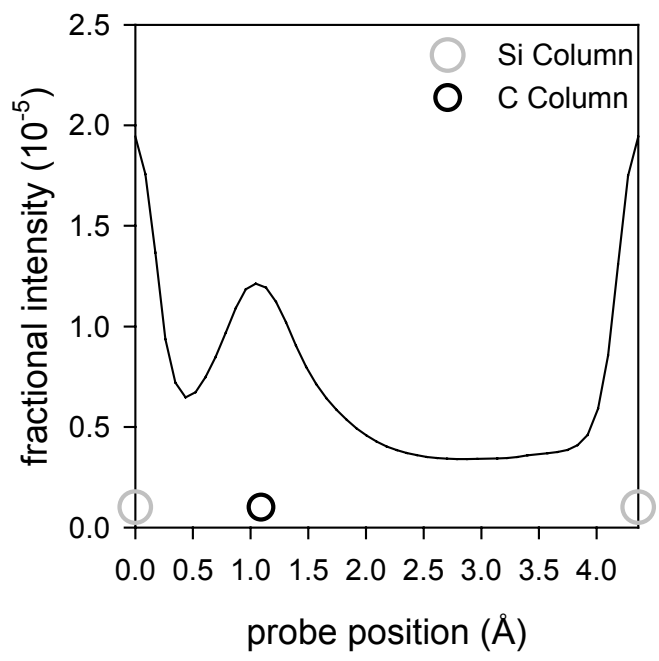

FIG. 2. Carbon $K$-shell EELS image in $\mathrm{SiC}$ for $200 \mathrm{keV}$ electrons incident along the [011] zone axis. The line scan is along the [100] direction. 